Revue d'histoire de l'Amérique française

PGS REVUE D.HISTOIRE DE L'AMÉRIQUE FRANÇAISE

\title{
La fondation du Collège militaire royal de Saint-Jean
}

\section{Jean-Yves Gravel}

Volume 27, numéro 2, septembre 1973

URI : https://id.erudit.org/iderudit/303266ar

DOI : https://doi.org/10.7202/303266ar

Aller au sommaire du numéro

Éditeur(s)

Institut d'histoire de l'Amérique française

ISSN

0035-2357 (imprimé)

1492-1383 (numérique)

Découvrir la revue

Citer cet article

Gravel, J.-Y. (1973). La fondation du Collège militaire royal de Saint-Jean. Revue d'histoire de l'Amérique française, 27(2), 257-280.

https://doi.org/10.7202/303266ar d'utilisation que vous pouvez consulter en ligne.

https://apropos.erudit.org/fr/usagers/politique-dutilisation/ 


\section{LA FONDATION DU COLLĖGE MILITAIRE ROYAL DE SAINT-JEAN}

Jean-Yves Gravel

Au pays de l'unité dans la diversité, les débats politiques, après coup, sont habituellement l'occasion d'une salutaire réflexion sur la société canadienne à l'œuvre et à l'épreuve. Le débat relatif à la fondation du Collège militaire de Saint-Jean, en 1952, permet une analyse touchant les rapports entre les Canadiens anglais et français ainsi que les relations civilesmilitaires. C'est le gouvernement, en qui s'affrontent les groupes de pression et l'opinion publique, qui doit alors trouver le moyen terme entre le progrès social et la mentalité collective, dilemme que compliquent les composantes ethniques en cause. Le compromis qui en résulte souvent marque une meilleure compréhension mutuelle. Le Collège militaire royal de Saint-Jean est l'un des nombreux compromis de la politique canadienne. C'est le compromis entre les Canadiens français qui réclament, au nom de l'unité nationale, une plus grande représentativité dans les Forces armées, et les Canadiens anglais pour qui toute concession au Québec menace cette même unité nationale. L'on utilise les mêmes mots, mais leurs racines se nourrissent dans un passé lourd de divergences.

\section{Situation des francophones}

Si les Canadiens français forment $29 \%$ de la population canadienne à l'époque de la guerre de Corée, leur représentation dans l'organisation militaire du pays est beaucoup moins élevée ${ }^{1}$. Dans la force permanente des trois armées, ils forment $6.9 \%$ des officiers et $15.3 \%$ des hommes de troupe. Du côté des promotions, les officiers francophones se trouvent dans les grades subalternes ${ }^{2}$ alors qu'ils ne comptent que $3.8 \%$ des 662 lieutenants-colonels ou l'équivalent, $1.2 \%$ des 162 colonels et deux

${ }^{1}$ ND Rec Stats, 15 octobre 1951, dans Archives Publiques du Canada, Claxton Papers, vol. 94: séries Q et V.

${ }^{2}$ Liste nominative de tous les officiers francophones par arme et grade, dans Claxton Papers, vol. 94: H.

\section{[257]}

RHAF, vol. 27, no 2 (septembre 1973) 
des 89 généraux. Ils sont aussi absents des centres de décision ${ }^{3}$. $\mathrm{Au}$ Grand Quartier Général (GQG), à Ottawa, ils sont doublement sous-représentés avec 55 officiers (soit $3.9 \%$ ) qui, pour la plupart, remplissent des fonctions sans importance. Au Québec même, ils n'occupent que quinze des 84 postes supérieurs.

Toutes les écoles de formation militaire fonctionnent en anglais seulement, sauf l'école du soldat du Royal 22e Régiment. Les Canadiens français qui s'enrôlent ont le choix de parler français tout en demeurant de simples fantassins, ou bien d'apprendre l'anglais pour monter en grade ou faire partie d'un corps technique. A plus forte raison dans la Marine et l'Aviation. Après un cours d'anglais fort élémentaire, les soldats francophones sont envoyés dans les écoles de métiers militaires situées en dehors du Québec. Pas pour longtemps. S'ils ne sont pas retournés à l'école d'anglais, ils ont de fortes chances de rater leur cours de métier tout en vivant dans une atmosphère hostile, sujets à l'ostracisme et aux préjugés ${ }^{4}$. Les instructeurs anglophones ne semblent pas comprendre que la recrue francophone doit, comme n'importe quel de ses compatriotes anglophones, s'adapter à la vie militaire, et, lorsque postée en dehors du Québec, s'habituer à d'autres coutumes, s'ajuster à une mentalité différente, tout en apprenant une langue étrangère. L'Armée se voit bien obligée de reconnaître son échec quant à l'intégration des soldats francophones non fantassins, surtout dans l'artillerie et les blindés.

C'est pour étudier cette question qu'un comité est formé en juillet 1950, sous la présidence du général de brigade Paul Bernatchez, alors le seul officier supérieur francophone à Ottawa. Jusqu'à sa retraite, en 1964, le général Bernatchez n'a cessé de travailler en faveur des Canadiens français en employant le langage direct qui le caractérise. Tant auprès des chefs de l'Etatmajor de l'Armée que des ministres de la Défense qui se sont succédé, il a préconisé des changements dans l'organisation militaire qui, tout en assurant l'efficacité opérationnelle, donneraient aux Forces armées canadiennes un caractère plus national. Ces réformes devaient favoriser un recrutement suffisant dans le Québec ainsi qu'une représentation francophone équitable à tous les niveaux.

3 Ibid.: C (1951 et 1952).

4 Bishop à Graham, 22 et 25 juillet 1950; Clift au DCGS, 3 août 1950; Bernatchez à Sparling, le 10 février 1951, dans PARC, Army Headquarters Files; "Notes on Language Problem Situation During the Past Year", 12 octobre 1951, dans Claxton Papers, vol. 94: E. 
Le Rapport Bernatchez ${ }^{5}$ est prêt en février 1951. La recommandation la plus importante est la création au Québec d'un camp d'instruction destiné à recevoir des sous-unités françaises pour toutes les armes. La langue de travail et de commandement y sera le français, pourvu que les officiers et les signaleurs soient bilingues afin d'assurer les communications avec les autres corps et les formations supérieures. Ces unités francophones s'imposent d'autant plus que les Canadiens français, qui ne forment que $18.4 \%$ de l'effectif de l'Armée, sont sousreprésentés dans les blindés avec $6.1 \%$, l'artillerie avec $6.8 \%$ et le génie avec 9.4\%. En outre, onze des quinze écoles de métiers n'ont aucun instructeur bilingue. Cela ne veut pas dire que les autres écoles donnent des cours dans les deux langues.

Le rapport tente aussi d'expliquer pourquoi les Canadiens français ne s'enrôlent pas. Ils croient que les Forces armées sont des institutions anglaises et que leurs possibilités d'emploi et de carrière sont déterminées par leur degré de bilinguisme. Ils savent qu'il n'est pas facile de devenir de parfaits bilingues et ils estiment en conséquence que la barrière linguistique nuira toujours à leur promotion, sauf s'ils servent dans des unités francophones comme le Vingt-Deuxième. Certains jeunes gens aimeraient bien s'enrôler ailleurs que dans l'infanterie, mais cela est pratiquement impossible s'ils ne sont pas bilingues. Encore imprégnés d'une mentalité régionale, ils hésitent à s'enrôler dans des corps comme l'artillerie et les blindés où ils devront passer la majeure partie de leur vie en dehors des centres francophones. Les autorités religieuses et de nombreux parents craignent que le service militaire dans des communautés à prédominance anglaise et protestante entraîne la perte de la langue et de la foi ; après quelques années dans la carrière, ce déracinement culturel et cette assimilation s'étendent à la famille du militaire francophone obligée de vivre en dehors du Québec, avec l'insoluble problème scolaire des enfants. Certains croient aussi à la discrimination directe contre les francophones. Enfin le faible recrutement vient de ce que les parents considèrent encore les Forces armées comme un endroit de réforme pour les fils récalcitrants au lieu d'y voir une véritable carrière militaire. L'Aviation, avec son image technique, sera la première des trois armes à briser ce mythe. Toutes ces croyances ont d'autant plus de poids que la vie militaire est quasi inconnue au Québec tout en étant impopulaire. Pour la plupart des gens, la Défense

5 Committee for the Study of Bilingual Problems, Conclusions and Recommendations, 12 p., dans Claxton Papers, vol. 94: B. 
nationale apparaît davantage une agence de bien-être social qu'un employeur.

L'Etat-major ne donne pas suite à l'essentiel du Rapport Bernatchez. Avant même d'en connaître les conclusions, le ministre de la Défense, Brooke Claxton, déclarait aux Communes que des sous-unités françaises au Québec étaient "peut-être désirables, mais impossibles économiquement" ". Un an plus tard, il précise le motif de son refus: avec des unités françaises, l'Armée serait plus que jamais composée de deux éléments ethniques, ce qui serait contraire à l'unité nationale et pourrait donner aux francophones l'impression d'être confinés à des ghettos ? ${ }^{7}$ Une académie militaire au Québec est précisément l'une de ces unités françaises auxquelles s'opposent le ministre et l'Etat-major.

La Marine éprouve, elle aussi, de sérieuses difficultés pour recruter des Canadiens français. En 1951, ils ne forment que $2.2 \%$ des officiers et à peine $11 \%$ des marins ${ }^{8}$. Sitôt plongés dans la grande tradition navale du HMCS Cornwallis, un navire au sol, les recrues francophones prennent en moyenne 38 semaines au lieu des 21 requises pour compléter leur cours de recrue dont l'atmosphère est propice au découragement. Le commandant Marcel Jetté est alors chargé d'évaluer la situation. A la fin de 1952, son rapport donne naissance au HMCS D'Iberville, une école située dans la Vieille Capitale et dont le cours de six mois prépare les recrues francophones pour Cornwallis.

Le Rapport Jetté ${ }^{9}$, l'un des plus intéressants sur la mentalité des Québécois d'après-guerre, analyse les raisons pour lesquelles ils ne s'enrôlent pas dans la Marine royale canadienne qu'ils confondent d'ailleurs avec la Marine marchande. Avant tout, les Canadiens français considèrent les marins comme des "bums" ( sic) avec une fille dans chaque port. Ils ont l'impression que la Marine est beaucoup plus britannique que canadienne, ce que confirmait le Rapport Mainguy ${ }^{10}$ publié deux ans plus tôt, et la croyance que le Canada participe à des guerres dont l'Angleterre est plus ou moins responsable. Les Canadiens français pensent aussi qu'ils ne seront pas promus en raison de leurs

6 Debates, 10 mai 1951, 2884.

7 "Two Languages in the Armed Services of Canada", p. 12a, dans Claxton Papers, vol. 94: V.

8 Claxton Papers, vol. 94: C, Q et V.

9 Report on Recruiting of French Speaking Canadians, au Directorate of History (Ottawa), $36 \mathrm{p}$.

10 Rapport Mainguy (Ottawa, Impr. du Roi, 1949), 62 p. 
difficultés linguistiques; c'est un fait que les marins francophones doivent d'abord apprendre l'anglais en peu de temps, puis maîtriser le jargon de la Marine qui est une autre langue en soi, à quoi s'ajoute l'obligation de vivre toute leur vie dans une mentalité distincte de leurs aspirations. En outre les liens familiaux sont encore forts au Québec; les mères, en particulier, n'aiment pas voir leurs fils partir pour une "province étrangère", encore moins vers des pays lointains dont les ports menacent le salut de leurs âmes. Si, malgré tout, le Canadien français se laisse apprivoiser par les recruteurs de la Marine, il doit d'abord réussir le test d'aptitudes (G.F. Test) uniquement adapté à la mentalité anglophone. C'est pourquoi $80 \%$ des Québécois ratent ce test qu'on a simplement "traduit" en français; le taux d'échecs chez les anglophones est de 52\%. L'impression générale est que la Marine ne veut pas de Canadiens français dans ses rangs, ce qui est un peu vrai si l'on songe qu'il n'y a que deux centres de recrutement dans la province comparativement aux sept centres en Ontario.

Etant la plus jeune des trois armes, l'Aviation aurait dû être la plus représentative de la réalité canadienne. C'est pourtant elle qui affiche le refus le plus catégorique au Canada français et au bilinguisme ${ }^{11}$. L'Aviation est d'abord matériellement absente du Québec, ce qui n'est pas sans conséquences économiques pour la province. En 1951, il n'y a qu'un seul QG de Commandement, d'ailleurs mineur, à Saint-Hubert, alors que l'Ontario détient les trois plus importants; aucune des dix bases d'entraînement ne se trouve au Québec. Les Canadiens français sont aussi absents de l'Aviation. Ils ne forment, en 1951, que $4.7 \%$ des officiers et $16.3 \%$ des aviateurs ${ }^{12}$. Le francophone le plus haut gradé est un colonel. Et à Saint-Hubert même, il ne se trouve aucun Québécois aux 37 postes de direction. La langue anglaise est naturellement omniprésente et exclusive en vertu du mythe que l'Aviation doit fonctionner en anglais par convention internationale. On ne distingue pas la tour de contrôle (moins de 1\% du personnel) des hangars et de l'administration.

L'adaptation des aviateurs québécois à un monde si différent en raison de la langue et de la technique ne se fait pas sans heurts ${ }^{13}$. Comme la recrue anglophone qui doit passer de la vie civile à la société militaire, la recrue francophone doit aussi

11 J. Eayrs, In Defence of Canada (U.T.P., 1972), III : 129.

12 Claxton Papers, vol. 94: C, Q et V.

13 H. C. Forbell, Armed Forces Historical Study (Commission B \& B, rapport no 20), Part III: 29. 
s'ajuster à une communauté étrangère. Elle doit en outre affronter trois autres difficultés au cours de son entraînement: apprendre la langue en peu de temps, se mesurer aux autres en anglais (instruction, manuels et examens), c'est-à-dire qu'elle est jugée sur le même pied que ceux dont c'est la langue maternelle; il se pose enfin un grave problème de motivation, car cela oblige le francophone à passer, en moyenne, deux fois plus de temps à sa formation militaire. Et la vie de toute recrue, pleine de harassement et d'humiliation, n'a rien non plus pour relever le moral. Dans ces conditions, il n'est pas surprenant que le taux d'échecs soit de $50 \%$ pour les aéro-techniciens et de $80 \%$ chez les navigants. La moitié de ces taux est considérée normale chez les recrues anglophones. Les autorités de l'Aviation d'après-guerre n'ont vraiment fait aucun effort pour comprendre les difficultés des francophones. Elles les tolèrent à la condition qu'ils acceptent de s'angliciser et de s'assimiler. L'Aviation connaîtra dans les années 1950 plus de cas de discrimination que l'Armée et la Marine ensemble.

Si les jeunes Québécois ne semblent pas davantage intéressés par la carrière militaire dans les trois armes, c'est qu'ils n'y voient pas d'avenir. Les Forces armées leur semblent un domaine réservé où seuls les anglophones peuvent réussir. Cette image est encore plus vraie pour les officiers, car, de 1924 à 1964, les Canadiens français représentent moins de $8 \%$ du groupe ${ }^{14}$.

Le recrutement des officiers est assuré de quatre façons ${ }^{15}$. En 1951, la majorité des nouveaux officiers $(60 \%)$ entrent "directement" dans les Forces armées et se voient accorder un brevet à court terme de 5 ou 7 ans après un cours secondaire. La deuxième source $(20 \%)$ comprend les officiers choisis parmi les hommes de troupe. La troisième méthode est le programme universitaire pour les officiers de réserve (UNTD, COTC, RUS, etc.) dont seulement $10 \%$ embrassent la carrière militaire à la fin de leurs études. Il y a, enfin, les "vrais officiers", ceux qui viennent des collèges militaires, là où sont formés les futurs généraux de mer, de terre et de l'air.

Depuis 1948, et après une dure lutte d'influence, le Canada compte deux collèges militaires interarmes: le Royal Military

14 Stats Section (Navy), ND Stats (RCAF) et ND Pers Stats (Army), dans Claxton Papers, vol. 94: C, Q et V; et Commission B \& B, étude $\mathrm{n}^{\circ} 22$, app. $4: 7 \mathrm{~s}$.

15 Report of National Defence, 1952 (Ottawa, Impr. de la Reine, 1952), 14s. 
College, de Kingston, et Royal Roads, à Victoria. Aux deux endroits, la présence des Canadiens français est assez réduite: ${ }^{16}$

\begin{tabular}{|c|c|c|c|c|c|c|}
\hline & \multicolumn{3}{|c|}{ Demandes d'admission } & \multicolumn{3}{|c|}{ Acceptés } \\
\hline & Total & Frane. & $\%$ & Total & Frane. & $\%$ \\
\hline $1948-49$ & 428 & 58 & 13.5 & 185 & 21 & 11.3 \\
\hline $1949-50$ & 431 & 43 & 10.0 & 170 & 17 & 10.0 \\
\hline $1950-51$ & 319 & 43 & 13.5 & 154 & 10 & 6.5 \\
\hline $1951-52$ & 281 & 23 & 8.2 & 163 & 13 & 8.0 \\
\hline
\end{tabular}

Les 21 candidats acceptés en septembre 1948 ne sont plus que 4 en juin 1951; des 17 cadets de septembre 1949, il n'en reste que 8 en juin 1952, et ainsi de suite. En outre, les rares diplômés quittent la carrière l'un après l'autre, sitôt leur contrat terminé avec la Défense nationale. Le taux d'échec dans les collèges militaires et aux autres écoles d'officiers est deux fois plus élevé chez les francophones.

A ces raisons du faible recrutement des soldats de langue française, s'ajoutent d'autres considérations dans le cas des élèves-officiers. Les Canadiens français qui désiraient aller au collège militaire devaient connaître l'anglais de sorte que les cadets francophones généralement acceptés venaient des minorités de l'Ontario et du Nouveau-Brunswick. Les rares Québécois admis l'ont été parce que le règlement de sélection veut que la moitié des cadets soient choisis au prorata de la population des provinces, l'autre moitié selon les dossiers académiques ${ }^{17}$. Les parents n'aiment pas voir leurs fils quitter trop jeunes la demeure familiale pour l'étranger tandis que les finissants des collèges classiques, qui terminent en moyenne à 21-22 ans, sont trop âgés pour être acceptés. Les communautés enseignantes non plus ne sont pas enclines à recommander la carrière militaire ${ }^{18}$ parce qu'elle est synonyme d'assimilation, sans diplôme à la fin des études et qu'elle n'est pas très haut cotée dans la considération sociale. La grande différence qui existe entre le cours classique du Québec et le cours dit scientifique des autres provinces est une autre source de difficultés. Enfin, la tradition sportive, si importante pour la formation du caractère dans les institutions anglo-saxonnes, est beaucoup moins forte dans les maisons

16 Claxton Papers, vol. 94 : C.

17 Le Devoir, 18 avril 1952; et Report of National Defence 1952, 17. Cette politique d'admission est encore en vigueur.

18 Bernatchez à Sparling, 4 juillet 1951: et Macklin à Sparling, 8 mai 1951, dans Army Headquarters Files. 
d'enseignement du Québec. En ne recrutant pas les étudiants des collèges classiques, les Forces armées limitent leur potentiel d'officiers en nombre et en qualité. En fait, elles se privent ainsi de l'élite de la province de Québec.

Les autorités militaires savent depuis longtemps que les cadets francophones ont besoin d'un cours préparatoire. Les mettre en compétition directe avec les cadets anglophones, à Kingston et à Victoria, les désavantage injustement. Déjà en 1946, le Rapport Chesley avait recommandé une année spéciale ${ }^{19}$. Et lors du débat public pour la réouverture du Royal Military College de Kingston, le général Foulkes avait même proposé au ministre Claxton, le 20 février 1947, d'organiser à l'Université Laval une section du collège militaire à l'intention des aspirants du Québec ${ }^{20}$. Cela répondait aux vœux du député Bona Arsenault qui fut le premier, en 1945, à réclamer une académie militaire au Québec avec l'appui du journal Le Droit d'Ottawa ${ }^{21}$. Cette perspective, toutefois, fit peur au ministre qui estimait plutôt que les cadets n'avaient besoin que d'une année préparatoire en anglais et en mathématiques ${ }^{22}$. Néanmoins, le Royal Military College et Royal Roads ouvrirent à l'automne 1948 sans rien de particulier pour le Québec. On négligea aussi les recommandations du rapport de 1946 quant à la place des militaires francophones dans la réorganisation des Forces armées canadiennes. ${ }^{23}$ La réduction d'après-guerre ne rendait pas les Canadiens français indispensables pour remplir les contingentements. Bien au contraire, il fallait licencier près de $90 \%$ de l'effectif. L'existence même des deux collèges militaires restait incertaine. Et en général, la valeur des officiers était autant à la baisse que le moral des hommes de troupe.

\section{Les offres}

Le vaste programme d'expansion militaire de $\$ 5$ milliards, nécessité principalement par la guerre de Corée puis par l'OTAN ${ }^{24}$, surprend d'autant plus que la démobilisation avait

19 R. A. Preston, Canada's RMC. A History of the Royal Military College (U.T.P., 1969), 311. Cette recommandation venait du général Bernatchez.

20 "The Armed Forces College", 10 mars 1947, dans Army Headquarters Files.

21 Debates, 30 octobre 1945, 1629-1632; et Le Droit, 31 octobre 1945. 22 Claxton à Foulkes, 10 mars 1947, dans Army Headquarters Files. 23 "Summary of the 1946 Study", dans Claxton Papers, vol. 94: B.

${ }^{24}$ Canada's Defence Programme, 1951-52 (Ottawa, Impr. du Roi, 1952), 26-30. 
été radicale. Le gouvernement doit alors multiplier les efforts pour recruter environ 7,000 nouveaux officiers dont au moins le tiers devrait venir du Québec. C'est ainsi que le Ministère offre de payer la scolarité à tout étudiant de dernière année d'université qui désire s'enrôler par la suite.

L'Université Laval veut profiter indirectement de cette offre. Etant en période de développement, elle éprouve des difficultés financières par suite du refus du premier ministre Duplessis d'accepter les subventions fédérales à l'éducation. Le 8 mai 1951, l'Université propose au ministre Claxton d'organiser un cours de trois ans en sciences militaires après un B.A. classique ${ }^{25}$. Si les Canadiens francais ne deviennent pas officiers, c'est en partie parce que la carrière militaire ne leur a jamais été présentée de la même façon que les autres professions comme le droit, la médecine, le génie, l'enseignement, etc., avec un diplôme à la fin des études. Un baccalauréat en sciences militaires, dispensé par une institution prestigieuse, devrait corriger cette carence et donner plus de valeur, plus d'attrait à la carrière des armes. En d'autres termes, l'Université Laval deviendrait l'institution officielle pour la formation des officiers francophones tout en servant de bouclier moral aux Forces armées dans le Québec.

Le programme suggéré semble idéal pour la formation d'un officier moderne: sciences et mathématiques, relations internationales, histoire et géographie, psychologie, administration, langues secondes, etc. Au sujet du bilinguisme, les étudiants seront en mesure de comprendre des cours donnés en anglais à la fin de la première année et devraient être raisonnablement bilingues à la fin de leurs études. Le niveau du programme comprend les deux premières années de la Faculté de sciences et un an de culture générale et d'administration. Les candidats, choisis selon les critères de la Défense nationale, logeront à la Citadelle et seront assujettis à la discipline militaire. L'été, ils se joindront aux cadets des collèges militaires et aux autres élèves-officiers pour l'entraînement spécialisé. Ce projet, que proposent l'abbé Jacques Garneau et Mgr Maurice Roy, permettrait aux Canadiens français d'être sur le même pied que leurs compatriotes anglophones en ce qui a trait aux mathématiques et aux sciences en plus d'être bilingues. Leur formation serait aussi mieux équilibrée entre les sciences, les arts et l'administration. Ce programme à forte tendance "sciences sociales" semble avoir effrayé l'Etat-major qui y voyait d'ailleurs l'influence cléricale.

25 Garneau à Claxton, 8 mai 1951, dans Army Headquarters Files. 
A la demande du ministre, le comité des vice-chefs d'Etatmajor, celui des chefs d'Etat-major ainsi que le Conseil de la Défense étudient, tour à tour, en juillet et en septembre 1951, la proposition de l'Université Laval ${ }^{26}$. Si le projet reçoit un accueil sympathique de la part de l'Aviation, désireuse de refaire son image chez les francophones, l'Armée et la Marine, par contre, s'y opposent avec vigueur. Elles apportent deux objections majeures ${ }^{27}$. Le plan de Laval est le premier pas vers une académie militaire au Québec. Cette ségrégation selon la langue serait nuisible à l'unité nationale tout en produisant deux groupes d'officiers. Les autres universités pourraient se prévaloir de cet arrangement particulier avec Laval pour réclamer un traitement analogue. La Marine ajoute qu'avec une telle politique de ségrégation linguistique, les francophones pourraient même, un jour, oser demander de servir en français sur un navire de Sa Majesté! La vraie solution pour obtenir des officiers du Québec, selon les mandarins militaires, c'est une année préparatoire, avec subventions, pour permettre aux cadets francophones d'apprendre l'anglais et les sciences, et d'être ainsi en mesure de continuer à Kingston. Les trois comités se rallient finalement au "compromis" d'un cours préparatoire que dispenseraient les universités Laval et Montréal. C'est à peu près la proposition même du ministre Claxton, formulée trois mois plus tôt, en suggérant une nouvelle méthode de formation d'officiers pour les francophones ${ }^{28}$.

Sitôt le projet de l'Université Laval rendu public, le Collège Sainte-Marie de Montréal propose à son tour, en mai 1951, une académie navale pour les officiers du Québec dont a tant besoin la Marine royale canadienne ${ }^{29}$. Il s'agit d'un entraînement militaire parallèle et complémentaire aux études classiques. Ce programme regrouperait 30 Corps de "Marines" dans autant de collèges classiques que superviserait l'Académie navale de Sainte-

26 "Extracts from minutes of 16th meeting Vice chiefs of Staff Committee", 9 et 16 juillet 1951; "Extracts from minutes of Chiefs of Staff Committee", 6 et 25 juillet 1951; et "Extracts from minutes of 55th Meeting of Defence Council", 14 septembre 1951, dans Claxton Papers, vol. 94.

27 Outre les procès-verbaux cités plus haut, voir Bernatchez à Sparling, 4 juillet 1951 et la réponse, 6 juillet dans Army Headquarters Files; et Miller au DNT, 28 mars 1951, et Ellis au CNS, 5 juillet 1951, dans Navy Headquarters Files.

28 Claxton à Foulkes, 3 juillet 1951, dans Claxton Papers, vol. 94; Claxton à Mgr Roy, 27 juin 1951, dans Army Hearquarters Files; et brouillon d'une longue lettre de Claxton à Mgr Roy, 12 octobre 1951, préparé par les chefs d'Etat-major, dans Navy Headquarters Files. 29 Correspondance et plan dans Navy Headquarters Files. 
Marie tout en s'occupant de l'entraînement d'été en mer. Le père Dechêne, qui commande déjà le Corps de Cadets de la Marine des Jésuites, se fait le promoteur du projet et ne demande rien de moins qu'un navire. A Ottawa, les autorités de la Marine scrutent le plan soumis avec leur lorgnette. Elles y voient aussitôt que les futurs "Marines" du Québec, étudieront la manœuvre navale avec les manuels de la Marine française au lieu de ceux de la Royal Navy. Il n'en faut pas plus pour que l'Académie navale de Sainte-Marie fasse naufrage. D'ailleurs le projet n'est pas sérieux et vient davantage du Corps de Cadets que des autorités du collège.

Entre-temps, le gouvernement doit trouver des renforts pour son contingent spécial de Corée levé dans "le tâtonnement, la hâte et l'improvisation" et dont le tiers des 10,000 hommes s'avère des indésirables et des déchets sociaux ${ }^{30}$. Le général Simonds qui n'a rien appris du passé, songe même à la conscription ${ }^{31}$. Le Ministère inonde les maisons d'enseignement de publicité militaire, non plus traduite, mais entièrement "pensée en français"; M. Claxton adresse une lettre à tous les employeurs du Québec leur demandant de permettre aux employés de s'enrôler pour la Corée ou avec les troupes de l'OTAN en Allemagne ${ }^{32}$. Tous ces efforts ne sont pas inutiles puisque la contribution totale des Canadiens français à la guerre de Corée est de $25.4 \%$; elle avait été de $12.6 \%$ au cours de la Grande Guerre et de $19 \%$ lors de la Deuxième Guerre mondiale ${ }^{33}$. Dans les moments de crise, la Défense nationale semble découvrir les Canadiens français en fonction des effectifs dont elle a besoin.

Si le recrutement des hommes de troupe est critique, celui des officiers devient dramatique. Il manque tant d'officiers de langue française que l'on doit même envoyer 18 anglophones unilingues avec le Royal 22e Régiment ${ }^{34}$, causant plus de problèmes qu'ils n'en ont résolu. De 1950 à 1952, le nombre d'officiers dans les trois éléments (mer, terre et air) passe de 7,300 à

30 H. F. Wood, Singulier champ de bataille. Histoire officielle de l'Armée canadienne en Corée (Ottawa, Impr. de la Reine, 1966), 28 et 34; et B. Claxton, Memoirs, V: 1028, inédits dans Claxton Papers, vol. 222. 31 Simonds à Claxton, 9 mai 1951, dans Claxton Papers, vol. 100. Voir aussi l'excellent article de J. Granatstein, "Strictly on Its Merits: The Conscription Issue in Canada after 1945", dans Queen's Quarterly (été 1972) : 192-206.

32 Le Devoir, 16 avril 1952.

33 Sutherland à Claxton, 7 octobre 1951, dans Claxton Papers, vol. 94: C; et Army Headquarters Files, 4 août 1950.

34 H. F. Wood, op. cit., 180 s. 
$14,744^{35}$. C'est pourquoi le Ministère fait l'impossible pour varier les méthodes de formation d'officiers en vue de favoriser leur recrutement ${ }^{36}$. Une académie militaire au Québec, toutefois, ne fait pas encore partie du programme de recrutement.

\section{L'offensive}

Le nouveau Collège militaire royal de Saint-Jean sera le résultat des efforts communs de la presse, de l'Opposition conservatrice et de la Chambre de Commerce du Québec.

Léon Balcer, lieutenant de marine lors de la dernière guerre et député conservateur de Trois-Rivières, garde le mérite d'avoir relancé avec succès le débat pour la fondation d'une académie militaire au Québec. C'est le 9 mai 1951, aux Communes, qu'il lance son premier cri d'alarme ${ }^{37}$. Le 27 juin, il revient à la charge. Un collège militaire aidera au recrutement des francophones, favorisera l'unité canadienne et permettra aux Canadiens français de devenir compétents. "Nous ne voulons pas, dit-il, faire donner des promotions à des gens non qualifiés. Nous demandons seulement l'égalité de chance." ${ }^{38}$ Ce collège permettra aussi d'apprendre l'anglais progressivement, car personne ne nie la nécessité de connaître cette langue, dans le domaine des affaires comme dans la carrière militaire. $M$. Balcer continue sa campagne devant le Club Richelieu de Montréal, en montrant les difficultés éprouvées par les cadets francophones, à Kingston et à Victoria, qui doivent y "apprendre la langue anglaise en même temps que leur carrière", tout en étant jugés sur le même pied que les autres cadets $^{39}$. Enfin le 29 octobre, le député réclame à nouveau un collège militaire de même qu'un navire où la langue de travail serait le français ${ }^{40}$.

M. Balcer ne demeure pas seul dans son entreprise. Plusieurs autres députés l'appuient aux Communes ${ }^{41}$, le général George Pearkes, critique militaire officiel de l'Opposition, Louis-Philippe Picard, député de Bellechasse, et André Gauthier, député du Lac Saint-Jean. Le chef des conservateurs, le colonel George Drew,

35 CMR, Programme d'ouverture (1952) : 16.

36 Débats, 12 juin $1952,3355 \mathrm{~s}$.

37 Ibid., 9 mai 1951, 2929.

38 Ibid., 27 juin, 4905 et $4914-4916$.

39 Compte rendu détaillé de la conférence dans Le Devoir, 24 août 1951. Il fut remercié par Daniel Johnson.

40 Débats, 29 octobre 1951, 517.

41 Ibid., 27 juin 1951, 4920 et 26 novembre, 1348. 
parle aussi en faveur du collège lors du congrès de son parti, tenu à Montréal le 27 octobre 1951; il suggère même le nom de Cartier pour la nouvelle académie ${ }^{42}$. Le député Frédéric Dorion exige, lui aussi, devant le Barreau de Québec dont il est le bâtonnier, la création d'un collège qui permettra aux francophones d'obtenir une représentation plus équitable dans les postes supérieurs ${ }^{43}$.

Vient ensuite l'apport de la Société Saint-Jean-Baptiste, organisme qui inquiète les chefs militaires ${ }^{44}$. A l'occasion de son congrès annuel, la Fédération fait parvenir au premier ministre Louis Saint-Laurent un mémoire lui rappelant "qu'un ostracisme à peine voilé s'exerce encore au sujet de l'emploi du français dans les services publics, l'administration et l'Armée" ${ }^{45}$. La Société recommande, entre autres choses, que les unités francophones soient commandées en français. En outre, elle "prie instamment le gouvernement d'établir le plus tôt possible un collège militaire bilingue", soit à Ottawa, soit au Québec.

La presse francophone participe aussi à l'offensive en faveur d'une académie militaire. Les trois journaux de choc sont le Devoir, le Droit et l'Action. Chez les journalistes, il faut souligner le rôle déterminant de Pierre Vigeant et de Lorenzo Paré, tout au long de la campagne de presse en 1951 et 1952. La presse anglophone du Québec se montre également favorable aux revendications des Canadiens français, en particulier pour les commandements en français ${ }^{46}$.

Chaque incident attise le feu du débat sur les Forces armées. Deux cas allument presqu'un incendie: l'affaire Desrochers et le Camp Petawawa. En juillet 1951, le lieutenant d'aviation J.-J. Desrochers est licencié pour s'être opposé au régime du "Speak White" ${ }^{47}$. Ce cas symbolique déclenche aussitôt une campagne d'indignation dans le Québec. Selon le correspondant parlementaire du Devoir ${ }^{48}$, le cas Desrochers "résume de façon éclatante le régime de brimades et de persécutions que l'on inflige aux

${ }^{42}$ Le Devoir, 25 novembre 1952. L'article rappelle le rôle de M. Balcer pour l'obtention du collège.

43 The Gazette, 24 octobre 1951.

44 Megill à Bernatchez, 17 juin 1950, dans Army Headquarters Files. vol. $94: \mathrm{J}$.

45 Mémoire de la S.S.J.B., 26 juillet 1951, dans Claxton Papers,

46 The Montreal Star, 29 juin 1951; The Gazette, 22 juillet, 6 août et 14 novembre 1952; The Quebec Chronicle, 23 mai 1952.

47 Debates, 27 juin 1951, 4801-4804 et 5 nov. 1951, 770-772.

48 Le Devoir, 5, 9, 12, 16 et 19 juillet 1951. 
officiers et aux soldats du Québec ..." C'est surtout l'Etat-major qui subit les foudres du journal, "cet état-major francophobe, cette caste militaire imbue d'impérialisme culturel qui sabote l'unité nationale". Les Canadiens français s'enrôlent dans les Forces armées canadiennes comme dans une Légion étrangère! Le Devoir en conclut invariablement qu'un collège militaire s'impose plus que jamais pour introduire plus de bilinguisme dans la Défense nationale. Moins d'un mois plus tard, c'est l'Armée qui s'attire les boulets de la presse à propos d'une situation presque identique au cas Desrochers. On obligeait les francophones du Camp Petawawa à parler anglais en tout temps, même entre eux, à la chambrée et au mess. Cette fois-ci, c'est le Droit ${ }^{49}$ qui conduit l'attaque. Plus tard, c'est une histoire semblable à la base de l'Aviation à Winnipeg ${ }^{50}$, puis la "provocation" de l'Air Defence Command, à Saint-Hubert, dont la devise parait en latin et en anglais seulement ${ }^{51}$. Et les autres journaux francophones de reprendre en chœur.

$\mathrm{Au}$ lieu de s'en prendre au Ministère de la Défense nationale au gré des événements quotidiens, le chroniqueur parlementaire de l'Action catholique, Lorenzo Paré, publie plutôt une étude bien documentée sur "Les Canadiens français et l'organisation militaire" 52. Cette série d'articles est reprise en entier dans le Devoir et le Droit, et en partie dans presque tous les autres quotidiens et hebdomadaires du Québec. L'opinion publique devient d'autant plus sensibilisée à la chose militaire que le Ministère procède à une campagne publicitaire intensive pour recruter les centaines de soldats rendus nécessaires par la guerre froide.

Malgré les bons sentiments du ministre Claxton sur l'unité nationale, rappelle le journaliste Paré, la situation des francophones dans les Forces armées ne fait que se détériorer. Cette vague harmonie des deux cultures est davantage fondée sur la rhétorique que sur la réalité. On veut intégrer les Canadiens français au système plutôt que de le modifier en partie pour répondre à leurs aspirations. Les jeunes militaires du Québec sont expatriés dans des centres entièrement étrangers sinon

49 Le Droit, 7, 8 et 11 août 1951. Les faits sont confirmés par Graham à Macklin, 7 août 1951 et par le rapport spécial de Bernatchez à Simonds, 11 août 1951, dans Claxton Papers, vol. 94: D.

50 Les aviateurs n'avaient pas le droit de parler en français à la base de Winnipeg, Le Devoir, 14 octobre 1952; faits confirmés dans un message du 2 octobre 1952, RCAF Headquarters Files.

51 Le Droit, 13 mai; L'Action, 8 mai 1952.

$52 \mathrm{La}$ série est publiée sous forme de brochure en octobre 1951 par l'CEuvre des Tracts de Montréal (n $\left.{ }^{\circ} 382\right), 16$ p. 
hostiles à leur langue et à leur mentalité. En fait les militaires québécois sont d'éternels exilés dans leur propre pays. Le gouvernement peut parler avec éloquence et peut-être aussi avec sincérité de l'égalité ethnique, du bilinguisme, mais l'on dirait que les galons dorés et les fonctionnaires font tout pour saboter l'unité canadienne.

M. Paré analyse ensuite la situation des collèges de Kingston et de Victoria. Il montre d'abord comment ces institutions contribuent à créer une caste de privilégiés dans les rangs militaires grâce à la protection mutuelle. Quoique formant une minorité, ces officiers jouissent d'une poussée initiale dans la carrière, d'avantages financiers et d'une priorité dans les promotions qui constituent une source d'injustice pour les "officiers civils" venus des universités et les gradés promus officiers. Ces deux collèges sont en outre une cause d'inégalité entre les provinces. En raison de la proximité de Royal Roads, les provinces de l'Ouest et du Pacifique fournissent quatre fois plus d'aspirants que les Maritimes qui n'ont droit qu'à six cadets. A Kingston, c'est naturellement l'Ontario qui a l'hégémonie complète. Ainsi ces deux académies dites "nationales" sont en fait "régionales" par leur recrutement. L'Est du Canada est donc en droit d'obtenir lui aussi son collège militaire.

De tous les groupes de pression qui ont travaillé pour l'académie militaire, la Fédération des Jeunes Chambres de Commerce est de loin l'organisme qui a joué le rôle le plus dynamique. Au début de 1952, la Fédération entreprend une campagne systématique d'information populaire et d'action politique pour intéresser les Canadiens français à la carrière militaire et pour faire lever les obstacles qui nuisent à leur avancement ${ }^{53}$. A cette intention, la Fédération écrit à tous les députés et journaux du pays pour leur demander leur opinion sur un collège militaire au Québec. Le comité, que dirige avec beaucoup d'efficacité Jean Vallée, de Québec, fait aussi préparer une série d'articles sur l'organisation militaire à l'intention de Jeune Commerce, revue officielle de l'organisme. Le Devoir et le Droit reproduisent en entier cette seconde série d'articles de Lorenzo Paré tandis que les autres journaux francophones en publient de larges extraits. La Chambre de Commerce favorise "une politique de présence": peuplons la base si nous voulons parvenir au sommet.

53 Historique du programme d'action, dans Le Droit, 21 juin 1952; L'Action et Le Soleil, 7 mars 1952; voir aussi Claxton Papers, vol. 94: U; et Vallée à Claxton, 9 février 1952, dans Army Headquarters Files. 
Le débat pour cette académie militaire arrivait au bon moment ${ }^{54}$. Grâce au nouveau principe des collèges interarmes, accepté en 1948, un seul collège suffirait pour les Canadiens français. Auparavant, il en aurait fallu un pour chaque élément. La campagne profita aussi du caractère "civil" des collèges militaires canadiens. A titre de compromis, Kingston et Royal Roads avaient pratiquement enlevé du programme académique tout ce qui était naval et militaire. Ils devenaient ainsi des universités civiles avec des étudiants en uniformes.

Ce concept canadien d'un collège militaire civil diffère fondamentalement de celui des autres pays. Même West Point, le plus "intellectuel" des collèges militaires, enseigne la tactique; il possède aussi un important département d'art et de génie militaires. Kingston et Royal Roads ont bien un module d'études militaires, mais il est à part. Cette révolution en éducation militaire, comme l'a appelée le professeur George Stanley, fut critiquée par des officiers réguliers qui préféraient le système traditionnel de la "fabrique des officiers" à la méthode prussienne. En fait, la population canadienne ne tolère les collèges militaires qu'en raison de leur caractère civil et que parce qu'ils peuvent fournir des diplômés utiles à la société.

\section{La défensive}

Divers groupes se sont opposés à la fondation du Collège militaire royal de Saint-Jean. Le projet rencontra successivement l'opposition du ministre de la Défense nationale, du chef de l'Etat-major de l'Armée, de la presse anglaise en dehors du Québec, mais surtout la résistance du R.M.C. Club.

S'il est une idée qui revient sans cesse dans les papiers du ministre Brooke Claxton ou dans ses mémoires inédits, c'est celle de la "canadianisation" des Forces armées ${ }^{55}$. Tant dans ses discours que par les décisions administratives, le ministre s'est fait l'avocat des intérêts du Canada que symbolisent la reconnaissance de l'hymne "O Canada" et du "Red Ensign" par les militaires, le Rapport Mainguy qui montra les difficultés du réajustement d'après-guerre et qui libéra la Marine de plusieurs traditions de Nelson déformées par l'usage, et le fait qu'il fallut

54 Cet intéressant commentaire m'est fourni par le lt-col. (mer) W. A. B. Douglas dans ses notes sur le manuscrit. Voir aussi George Stanley, "Military Education in Canada, 1867-1970", dans H. J. Massey, The Canadian Military, a profile (Toronto, Clark, 1972), 178-184.

55 Claxton Papers, vol. 94: A, M et $Q$; et vol. 100: 3-10. 
forcer les militaires canadiens à porter l'écusson 'Canada' sur leurs épaules. Le ministre associait à cette canadianisation le caractère bilingue du pays. Il n'a jamais manqué une occasion de promouvoir le bilinguisme ou de promettre l'égalité de chances aux militaires francophones ${ }^{56}$. Toutefois, malgré une bonne volonté évidente et de sincères efforts, cet aspect de sa politique est demeuré au niveau de la rhétorique que dénonçait la presse du Québec.

Il semble que l'Etat-major ait saboté le programme du ministre. Paul Mathieu, sous-ministre adjoint, écrivait avec regret: "J'ai l'impression que les instructions du ministre n'ont pas toujours atteint les officiers subalternes qui exécutent le travail ${ }^{57}$." En février 1952, par exemple, Claxton suggérait une série de mesures pour donner plus de justice aux Canadiens français ${ }^{58}$ : cours et examens dans leur langue, ordres bilingues, etc. Ces recommandations ne descendirent pas plus bas que le général Simonds qui signifia au ministre une fin de non recevoir ${ }^{59}$. Le ministre n'insista pas. En une autre occasion, Claxton adressa une lettre sévère à l'Aviation pour avoir mené au Québec, en anglais seulement, une campagne de recrutement, et cela malgré les ordres formels à ce sujet ${ }^{60}$. Peu de temps après, l'Etat-major fit préparer par des publicitaires une brochure sur "le bilinguisme dans les Forces armées". Chef-d'œuvre d'équivoque et de démagogie, c'était en fait un énergique plaidoyer pour justifier l'usage exclusif de l'anglais dans la vie militaire canadienne. Il fallut au ministre Claxton toute son autorité pour empêcher la publication d'une brochure si provocatrice ${ }^{61}$. Une fois même, le ministre réunit tous les officiers du GQG d'Ottawa et leur servit une mercuriale pour qu'ils s'éveillent à la réalité québécoise ${ }^{62}$. Non sans raison, les journaux francophones ont maintes fois accusé les officiers supérieurs d'être "des saboteurs de l'unité nationale".

56 Claxton à Simonds, 29 février 1952, dans Claxton Papers, vol. 94. 57 Mathieu à Blanchette, 8 août 1951, dans Claxton Papers, vol. 94: M. 58 Claxton à Simonds, 29 février 1952, dans Claxton Papers, vol. 94.

59 Simonds à Claxton, 5 mars 1952, dans Army Headquarters Files. 60 Claxton aux Chefs d'Etat-major, 6 décembre 1950, dans $R C A F$ Headquarters Files.

61 "The Two Languages in the Armed Services of Canada", 25 p., dans Claxton Papers, vol, 94: V.

${ }_{62}$ Le Devoir, 5 octobre 1951. Il y a quelques temps, l'amiral E. R. Zumwalt blâma sévèrement son Etat-major pour avoir bloqué son programme d'égalité raciale dans la Marine américaine, Christian Science Monitor, 13 novembre 1972 et U.S. News \& World Report, 27 novembre 1972, 27-31. 
Que le ministre ait été victime ou non des préjugés de ses conseillers militaires, ses efforts en faveur des Canadiens français n'ont guère dépassé les discours de circonstance. Les deux seules cuvres durables qu'il accomplit en ce domaine sont la formation des $2 \mathrm{e}$ et $3 \mathrm{e}$ Bataillons du Vingt-Deuxième, décision dictée par la Guerre froide, et la fondation du Collège militaire de Saint-Jean qui lui a été imposée par l'opinion publique du Québec.

Pendant plus d'un an, le ministre Claxton s'était opposé d'une façon personnelle et catégorique au projet. Son refus reposait essentiellement sur sa conception de l'unité nationale: "Nous ne saurions, disait-il, avoir deux systèmes d'écoles militaires et deux groupes d'officiers ${ }^{63}$." D'ailleurs une telle dépense ne serait raisonnable qu'en temps de guerre ${ }^{64}$. Les journaux lui rappellent aussitôt qu'on avait, au contraire, fermé le Royal Military College pendant la Deuxième Guerre mondiale. Attentif aux scandales qui ébranlent l'Académie militaire de West Point, aux Etats-Unis ${ }^{65}$, le ministre n'en devient que plus sceptique au sujet des collèges militaires ${ }^{66}$. De fait, nul ministre de la Défense ne parle autant en faveur de la formation des officiers dans les universités civiles. Dans les cercles militaires et politiques, on mène une campagne pour expliquer que les collèges militaires sont des institutions désuètes ou des établissements de luxe ${ }^{67}$. On prétend aussi qu'un collège unilingue français desservirait la cause des francophones qui ont besoin de bien connaître l'anglais s'ils veulent avancer dans leur carrière. D'ailleurs il serait quasi impossible de trouver le nombre suffisant de professeurs parmi les officiers français des trois éléments ${ }^{68}$.

Tandis que le ministre Claxton s'oppose au projet, surtout au nom de l'unité canadienne, son chef d'Etat-major de l'Armée, le lieutenant-général Guy Simonds, s'y oppose aussi, mais au nom de l'efficacité militaire. Dans un mémorandum "d'avertissement", il précise au ministre qu'avec les ordres en français lors des cérémonies publiques au Québec, le ministère est allé "à la limite" de ce qui peut être fait pour le bilinguisme ${ }^{69}$. Pour des

63 Le Devoir, 15 mars 1952; Débats, 27 juin 1951, 4915.

64 Débats 10 mai 1951,2950. Kingston coûte $\$ 887,000$ par année et Victoria $\$ 666,000$, Débats, 27 juin 1951, 4915.

65 The Montreal Star, 10 août 1951.

66 Voir ses Memoirs, V : 986-998; et Le Droit, 15 mars 1952.

${ }^{67}$ Le Devoir, 16 et 23 avril ainsi que 29 mai 1952.

68 "The Languages ..." 21, dans Claxton Papers, vol. 94: U.

69 Simonds à Claxton, 5 mars 1952, dans Army Headquarters Files. 
raisons d'efficacité militaire, poursuit Simonds, on doit s'opposer à la "minorité extrémiste" qui désire cette académie francophone. Un tel projet serait "fatal à l'unité nationale". Le général reconnaît cependant la nécessité d'une année préparatoire pour les cadets de l'Est du Canada. Néanmoins, placer cette institution à Québec même serait une grave erreur, car les militaires en perdraient bientôt le contrôle sous "l'influence dominatrice" de l'Université Laval.

Derrière le ministre et l'Etat-major se cache le "R.M.C. Club", le véritable adversaire du projet. Groupant tous les diplômés du Royal Military College of Canada, cette association forme une sorte de petite mafia aussi appelée "the RMC oldboy network" " ${ }^{0}$. Encore aguerri par sa victoire pour la réouverture de Kingston, le R.M.C. Club donne l'alerte sitôt le projet lancé. Cette nouvelle académie militaire menace l'unité nationale et met en danger l'efficacité militaire; mais surtout, elle risque de diminuer l'influence et l'importance de leur alma mater. Le colonel Reginald Sawyer, directeur des études à Kingston, fut le principal opposant et le conseiller le plus convaincant de Claxton et de l'Etat-major.

Les journaux anglophones sont les alliés naturels du ministre dans sa campagne contre le projet. La presse reprend les arguments du ministre Claxton ou ceux du général Simonds. Selon le Brandon Sun du 4 juin 1952, cette académie retarderait davantage l'unité canadienne et limiterait les Québécois à leur "paroisse". Le bilinguisme dans les Forces armées est impraticable et mène à la confusion. Même opinion dans l'Ottawa Citizen du 20 mai qui s'oppose au collège au nom des "faits"; que ce soit désirable ou non, les Forces armées canadiennes doivent fonctionner en anglais, langue prédominante dans le monde. Ce qu'il faut aux Canadiens français, ce sont des écoles d'anglais pour leur fournir l'égalité de chances qu'ils réclament. Pour le Globe and Mail du 4 juin, ce projet encourage "le séparatisme"; au lieu de briser la barrière des langues, il la renforcit. D'autres journaux, enfin, adoptent le genre équivoque "pas pour pas contre". La presse anglophone est caractéristique du point de vue d'un grand nombre de Canadiens anglais qui, tout en admettant en théorie le bilinguisme, agissent en niant pratiquement l'usage du français. La seule exception heureuse est le Winnipeg Free Press qui a fait preuve d'une compréhension remarquable des problèmes que doivent affronter les militaires francophones.

${ }^{70}$ Voir leur bataille pour la réouverture du collège, dans R. A. Preston, Canada's RMC, 305-331. 


\section{La politisation du projet}

La campagne en faveur de l'académie militaire prend une dimension nouvelle au printemps 1952 avec les élections partielles fédérales. Les conservateurs tiennent à gagner les comtés de Brome-Missisquoi et de Roberval comme tête de pont au Québec avant les élections générales prévues pour l'année suivante. Nul doute que les conservateurs, y inclus M. Balcer, ont exploité le projet du collège militaire, en 1952 , moins par conviction que pour embarrasser le gouvernement ${ }^{71}$. Toutefois, ce ne fut que l'un des thèmes électoraux parmi bien d'autres.

Les conservateurs mènent le combat en Chambre et dans la province. C'est d'abord le major George Hees, lui-même un diplômé du R.M.C. en 1931, qui ouvre le feu en se prononçant en faveur d'une telle académie pour le Québec ${ }^{72}$. Les journaux font un large écho à son discours que résume le Devoir en disant: "Il est un peu extraordinaire de voir un député torontois réclamer pour nous ce collège militaire français que nous refuse obstinément un ministre montréalais." ${ }^{73}$ Le 4 avril, Léon Balcer prend la relève. Pendant que son discours aux Communes lui vaut une manchette sur quatre colonnes ${ }^{74}$, il répète son message, cette fois, devant le Club Richelieu de Québec ${ }^{75}$. Et alors que la campagne électorale bat son plein, le chef de l'Opposition, George Drew, se prononce à nouveau dans les deux circonscriptions du Québec en faveur du collège ${ }^{76}$.

Les libéraux se doivent de réagir. Ils ont déjà perdu plusieurs comtés lors des élections partielles de 1949 et les augures pour 1952 ne sont pas trop encourageants. Devant l'expectative d'une nouvelle défaite, les députés libéraux du Québec pressent le gouvernement de faire quelque chose. Pourtant au cabinet, le ministre Hugues Lapointe, ancien combattant influent et respecté, défend sans relâche le projet avec l'appui du premier ministre Saint-Laurent ${ }^{77}$. A la suite de la lettre que la Chambre de Commerce a adressée à tous les députés ${ }^{78}$, les jeunes libéraux,

71 The Ottawa Citizen, 9 et 21 mai 1952.

72 Débats, 20 mars 1952, 664s.

73 Le Devoir, 25 mars 1952; Claxton était député de Saint-Laurent Saint-Georges. Voir aussi L'Action, 21 mars et Le Droit, 24 mars 1952.

74 Débats, 4 avril 1952,1208s.; Le Devoir et Le Droit, 5 avril 1952.

75 L'Action, $1^{\mathrm{er}}$ mai 1952.

76 Le Devoir, 29 avril; Le Soleil et The Quebec Chronicle, 19 mai 1952. cles, 132.

77 Le Devoir, 25 novembre 1952; et Bona Arsenault, Malgré les obsta-

78 Le Devoir, 6 et 15 mars et 12 avril 1952; Le Soleil, 7 mai 1952. 
réunis en caucus, forment un comité d'étude sur la "situation des Canadiens français dans les Forces armées". Ils sont d'autant plus furieux de l'inaction du gouvernement que les conservateurs appuient le projet du collège militaire. Par-dessus tout, le parti risque de perdre ces deux comtés du Québec.

Le ministre Brooke Claxton éprouve des moments pénibles de déchirement moral, partagé entre son opposition au collège avec l'appui de ses conseillers militaires et l'opinion publique du Québec travaillée par les groupes de pression et les journaux auxquels se joignent maintenant les députés libéraux de la province en plus des conservateurs. Claxton, qui a plus de trente ans d'expérience publique, connaît la grande règle de la politique: le compromis. Le Québec aura donc un mini-collège militaire qui dispensera une année préparatoire et qui sera ouvert tant aux cadets francophones qu'anglophones. Il s'agit maintenant de faire accepter le compromis à l'Etat-major et à la population du Québec.

Du côté de l'Etat-major, ce n'est pas difficile, car les officiers supérieurs sont depuis longtemps favorables à cette solution du "problème" des cadets francophones. Croyant pouvoir aider au recrutement des officiers dans la province, le major général Bernatchez, nouveau commandant de la Région militaire du Québec, resoumettait, en février 1952, le projet d'une année préparatoire d'anglais et de mathématiques que donnerait l'Université Laval ${ }^{79}$. Le Comité des Chefs d'Etat-major étudie la proposition et l'accepte le 15 mars, à titre de compromis. Les généraux Simonds et Bernatchez ainsi que le ministre Claxton se rendent aussitôt rencontrer les autorités de Laval. Mais l'Université n'est pas intéressée à donner cette année préparatoire pour le R.M.C. ${ }^{80}$ La Défense nationale doit en conséquence organiser elle-même ce cours spécial. Le Comité d'Etat-major accepte cette autre solution à sa réunion du 26 mai avec une prévision pour deux autres années si la demande le justifie. Une seule question demeure en suspens: faut-il installer le collège à la Citadelle ou à Saint-Jean? ${ }^{81}$

79 Bernatchez au VCGS, 9 février 1952, dans Army Headquarters Files.

80 Foulkes aux chefs d'Etat-major, 15 mars 1952, dans Army Headquarters Files et R. A. Preston, Canada's RMC, 345 .

$81 \mathrm{La}$ Citadelle n'étant pas jugée appropriée, on décida d'utiliser les casernes de Saint-Jean alors occupées par l'Ecole des recrues qui déménagea en vitesse" à Valcartier, Bernatchez au VCGS, 29 avril 1952, dans Army Headquarters Files; et Jean Berthiaume, "Les débuts du CMR", dans La Revue du CMR, 1952-53: 31-33. 
Dans le cas de l'opinion publique, le ministre veut d'abord tâter le terrain. Quoi de plus fertile que le Congrès de la Légion canadienne à Montréal où il promet de faire quelque chose pour assurer l'instruction militaire des Canadiens français ${ }^{82}$. Les journaux s'interrogent. La population attend sans trop comprendre. Les libéraux retiennent leur souffle électoral. Enfin, le 12 juin, quatre jours avant les élections, le ministre Claxton annonce la grande nouvelle aux Communes ${ }^{83}$. Le Québec aura un collège militaire ouvert à tous les Canadiens, avec la collaboration des universités Laval et de Montréal; et si le nombre de cadets est suffisant, l'on y donnera plus tard les première et deuxième années du cours régulier. Le nouveau collège sera placé sur le même pied que le Royal Military College et Royal Roads tandis que les cadets seront soumis aux mêmes conditions de service et porteront le même uniforme.

La réaction à l'annonce du ministre est positive dans l'ensemble. Même si le nouveau collège militaire n'est pas la véritable académie qu'ils ont réclamée, les journaux du Québec se montrent satisfaits ${ }^{84}$. Le Devoir et le Droit, qui auraient raison de se réjouir pour avoir mené le bon combat, sont beaucoup plus modérés. Pour le Droit, “ce n'est là qu'un premier pas, un jalon; la victoire finale est encore loin" ${ }^{85}$. Même réserve dans le Devoir qui estime que le ministre a adopté une solution de compromis pour éviter que les Forces armées canadiennes ne soient divisées par la question de la langue ${ }^{86}$. De tous les autres journaux français, seule la Patrie se particularise en insistant avec perspicacité sur le rôle d'employeur des Forces armées, sur les avantages de la carrière militaire en temps de paix et sur l'importance de la Défense nationale dans l'économie régionale ${ }^{87}$.

Chez la presse anglaise en dehors du Québec, la réaction dans l'ensemble en est une d'indifférence. Quelques quotidiens soulignent "avec satisfaction" la fondation du nouveau collège, mais glissent dans leurs éditoriaux quelques flèches acérées. Par exemple, pour le Peterborough Examiner du 16 juin, si le collège existe pour montrer l'anglais aux francophones, "well and good",

${ }^{82}$ Le Canada, 21 mai; The Quebec Chronicle, 23 mai; Le Soleil, 24 mai; et Le Devoir, 20 et 21 mai 1952.

83 Débats, 12 juin 1952, 3356.

84 Revue de la presse, dans L'Action, 19 juin et 31 juillet; Le Canada, 13 juin et 25 juillet; La Presse, 30 juillet 1952.

85 Le Droit, 21 juillet, 16 et 19 juin, 24 juillet et 20 août 1952.

$86 \mathrm{Le}$ Devoir, 30 juillet et 4 août 1952.

87 La Patrie, 23 juillet et 22 août 1952. 
sinon il est inutile. Selon le Victoria Colonist du 30 juillet, ce collège permettra aux cadets du Québec de rattraper leur "retard académique"! Le même jour, l'Ottawa Citizen se pose plutôt des questions: Comment le programme pourra-t-il combler l'écart entre le système d'éducation du Québec et celui de l'Ontario adopté par le R.M.C.? Comment fonder une nouvelle université en un mois? Comment, dans un domaine de juridiction provinciale, les autorités fédérales seront-elles capables d'obtenir la collaboration des éducateurs du Québec? Enfin, presque tous les journaux anglophones se font un devoir de rappeler que l'anglais doit rester la langue militaire, comme c'est le cas pour les affaires, l'industrie, les sciences, et ailleurs.

Dès l'annonce du nouveau collège militaire, tout se fait au pas accéléré. Les demandes de renseignements arrivent de partout. Plus de 1,000 lettres dont la majorité viennent du Québec et de l'Ontario ${ }^{88}$. Bientôt les demandes d'admission dépassent les prédictions les plus optimistes. Le ministre confirme aussitôt l'établissement d'une première et deuxième années et porte le nombre de cadets de 100 à $125^{89}$. Le 21 juillet, la nouvelle académie militaire est baptisée: Collège militaire royal de SaintJean ${ }^{90}$, tandis que le colonel Marcellin Lahaie en devient le premier commandant sous la recommandation personnelle du général Bernatchez. Les professeurs viennent presque tous du niveau collégial; quatre sont de l'Université de Montréal, mais aucun de Laval ${ }^{91}$; et à la demande du colonel Lahaie, le Royal Military College y envoie le major Léopold Lamontagne. Enfin tout est prêt. Le 13 novembre 1952, le ministre Claxton procède à l'ouverture officielle du collège. Pourtant, parmi les 500 hôtes d'honneur, on ne retrouve pas celui que tous les journaux surnommaient le parrain du collège. Léon Balcer n'avait pas été invité.

88 Sutherland à Claxton, 14 août 1952, dans Claxton Papers, vol. 94: V. 89 Débats, 25 juin 1952,3941 s.

90 Ce nom fut un autre compromis entre "Le Collège de Salaberry" et "Le Collège D'Iberville", R. A. Preston, Canada's RMC, 345.

91 Liste des diplômés, des publications et de l'origine des professeurs, dans La Revue du CMR, 1952-53:76-80 et 120s. Ils furent tous enrôlés comme officiers pour déjouer les lenteurs de la Fonction publique, ibid.: 32. Parmi les "pionniers" du CMR, nous retrouvons: $D^{r}$ Rosario Bilodeau, Me Denys Dion, général de brigade (Air) Daniel Gagnon, J.-Maurice Jarry, Hubert Laniel, $\mathrm{D}^{\mathrm{r}}$ Robert Lavigne, $\mathrm{D}^{\mathrm{r}}$ Bernard Ostiguy, Yvan Pigeon, Eugène-L. Roy et $\mathrm{D}^{\mathrm{r}}$ Donald E. Tilley. 
La résistance de l'Etat-major à la fondation d'une académie militaire au Québec était à l'image du Canada anglais. Psychologiquement on n'était pas prêt à accepter le bilinguisme que symbolisait ce nouveau collège, bilinguisme qui heurtait les préjugés collectifs et dérangeait les traditions politiques. L'anglais devait demeurer l'unique langue "militaire", réalité que même les francophones du milieu acceptaient ou toléraient. Ce collège ne signifiait aucunement la reconnaissance d'une seconde langue de travail, jugée une véritable menace à l'efficacité militaire. D'ailleurs la récente expérience de l'OTAN leur donnait raison. Dès le début, en effet, il fallut uniformiser les manœuvres, la théorie navale et les transmissions. Et le procédé ne faisait que commencer. L'efficacité militaire demandait la standardisation, une langue commune de travail et l'unité de commandement. L'Etat-major n'avait certes pas l'intention de modifier cet état de chose avec l'ouverture de l'académie militaire. Toutefois, le C.M.R. permettrait à plus de Canadiens français de faire une carrière militaire en leur facilitant l'entrée dans le système déjà existant des collèges militaires.

Sur le plan politique, l'opinion publique du Québec força le gouvernement à rendre les Forces armées plus représentatives de la réalité sociale canadienne. Le Collège militaire royal de Saint-Jean devait s'avérer l'une des rares institutions canadiennes vraiment typiques, de conception et de réalisation, ce que n'a jamais été le Royal Military College et ce que les Forces armées canadiennes tentent présentement de devenir, après un retard d'un siècle. 